\title{
On Distributed Source Coding Using Abelian Group Codes
}

\author{
Aria G. Sahebi and S. Sandeep Pradhan, \\ Department of Electrical Engineering and Computer Science, \\ University of Michigan, Ann Arbor, MI 48109, USA \\ email: ariaghs@umich.edu, pradhanv@umich.edu
}

\begin{abstract}
In this paper, we consider the distributed source coding problem with a joint distortion criterion. We use random structured codes, specifically nested Abelian group codes to achieve a new inner bound to the rate-distortion region. For certain sources, this inner bound is strictly larger than other known rate regions. This shows that structured quantization followed by structured binning is sufficient to achieve the BergerTung rate region. Furthermore, we define the notion of "nested random/group codes" in which the inner code is a group code and the outer code is a random code such that the quantization operation is a random vector quantization and the binning operation is structured. We use random/group codes to improve upon the rate region achieved using nested group codes. We define two fundamental quantities of Abelian group codes: the "channel coding group entropy" and the "source coding group entropy" to present the achievable rate region.
\end{abstract}

\section{INTRODUCTION}

In the general setup of the two-user distributed source coding problem, two encoders observe the realizations of two correlated sources and each sends a quantized version of their corresponding source sequences to a joint decoder. The decoder wishes to reconstruct a function of the two source realizations to within some distortion level. The goal is to find the rate pairs at which encoders can communicate with the decoder without exceeding a given distortion level. This problem has been studied extensively in the literature and there have been solutions for several special cases of this problem [2]-[8], [10], [11], [13], [18].

The best known inner bound to the achievable rate region using random codes is the Berger-Tung inner bound which is shown to be optimal for several special cases. In most of the existing coding strategies for this problem, each encoder performs sequentially two many-to-one mappings: a quantization mapping which maps several neighboring codewords to one codeword and a binning mapping which maps several widely separated codewords to a common index. The quantization mapping is typically taken to be a random vector quantization and the binning is a random independent indexing of the codewords. An exception is the work of Korner and Marton [6] in which the problem of reconstructing the modulo-two sum of two correlated binary sources is considered. In their work, the binning operation is a correlated indexing of the codewords using linear codes and it has been shown that using the structure of linear codes, correlated binning of the codewords can result in gains in terms of the achievable rate, for certain correlated sources. The work of Krithivasan and Pradhan [1] builds upon the work of Korner and Marton and provides a new achievable rate region for the general problem of distributed source coding using structured codes. This work relies on using nested group codes for encoding and uses a structured quantization followed by a correlated binning dictated by the structure of the code. In [1], the decomposition of Abelian group codes into cyclic groups is used to decompose codewords into several "digit" sequences and nested group codes are constructed for each digit of the code. The encoding and decoding operations are hence done successively for different digits of the codewords. The rate region provided by Krithivasan and Pradhan unifies all the known results on the distributed source coding problem and contains points which lie outside the other known inner bounds to the achievable rate region.

In the present paper, we present a new rate region for the distributed source coding problem using Abelian group codes. This rate region strictly contains the rate region provided by Krithivasan and Pradhan and benefits from several advantages. One advantage of the current work is gained by making the observation that in order to use the structure of the code to get rate gains, we do not require the quantization operation to be structured. In [1], both of the quantization and binning operations are taken to be structured operations based on group codes. However, only the correlated binning is necessary to get rate gains and the structured quantization operation causes a loss in terms of the achievable rate. In this work, we employ nested random/group codes so that the inner code is a group code and the outer code consists of several random cosets of the inner code. Hence, our quantization operation (related to the outer code) is random whereas our binning operation (related to the inner code) is correlated. Another difference of this work compared to [1] is that we build nested group codes directly on the Abelian group and not sequentially on its digits; hence, our encoding and decoding of the digits is done in a joint manner which is superior to the successive encoding/decoding scheme suggested in [1]. This also leads to a more compact description of the achievable rate region as compared to the one provided in [1]. 
The paper is organized as follows: In Section II, we formally define the problem and state known results for the problem. In Sections III and IV, we define several concepts which are used throughout the paper. In Section V, we consider the problem of reconstructing the sum of two correlated sources where the summation is the operation of some arbitrary Abelian group. We use this result in the proof of the coding theorem for the general distributed source coding problem. In Section VI, we employ nested group codes for the Wyner-Ziv problem and find an achievable rate region for this problem. In Section VII, we extend the result of Section VI to the distributed source coding problem. We state our coding theorem in Section VIII and we use nested random/group codes in Section IX to improve the rate region. In Section $\mathrm{X}$, we compare the new rate region with other known results and we conclude in Section $\mathrm{XI}$.

\section{Problem Definition and Known Results}

Consider two distributed sources generating discrete random variables $X$ and $Y$. Assume $X$ and $Y$ take values from alphabets $\mathcal{X}$ and $\mathcal{Y}$ respectively with joint distribution $p_{X Y}(\cdot, \cdot)$. The source sequence $\left(X^{n}, Y^{n}\right)$ is independent over time and has the product distribution $P\left(\left(X^{n}, Y^{n}\right)=\right.$ $\left.\left(x^{n}, y^{n}\right)\right)=\prod_{i=1}^{n} p_{X Y}\left(x_{i}, y_{i}\right)$. We consider the following distributed source coding problem: The two components of the source are observed by two encoders which do not communicate with each other. Each encoder communicates a compressed version of its input through a noiseless channel to a joint decoder. The decoder wishes to reconstruct the sources with respect to a general fidelity criterion. Let $\hat{\mathcal{Z}}$ denote the reconstruction alphabet, and the fidelity criterion is characterized by a mapping: $d: \mathcal{X} \times \mathcal{Y} \times \hat{\mathcal{Z}} \rightarrow \mathbb{R}^{+}$. We restrict our attention to additive distortion measures, i.e., the distortion among three $n$-length sequences $x^{n}, y^{n}$ and $\hat{z}^{n}$ is given by

$$
\hat{d}\left(x^{n}, y^{n}, \hat{z}^{n}\right) \triangleq \frac{1}{n} \sum_{i=1}^{n} d\left(x_{i}, y_{i}, \hat{z}_{i}\right) .
$$

In this work, we will concentrate on the above distributed source coding problem with one distortion constraint, and provide an information-theoretic inner bound to the optimal rate-distortion region.

Definition 1. Given a discrete source with joint distribution $p_{X Y}(x, y)$ and a distortion function $d(\cdot, \cdot, \cdot)$, a transmission system with parameters $\left(n, \theta_{1}, \theta_{2}, \Delta\right)$ is defined by the set of mappings

$$
\begin{gathered}
f_{1}: \mathcal{X}^{n} \rightarrow\left\{1, \ldots, \theta_{1}\right\}, \quad f_{2}: \mathcal{Y}^{n} \rightarrow\left\{1, \ldots, \theta_{2}\right\} \\
g:\left\{1, \ldots, \theta_{1}\right\} \times\left\{1, \ldots, \theta_{2}\right\} \rightarrow \hat{\mathcal{Z}}^{n}
\end{gathered}
$$

such that the following constraint is satisfied.

$$
\mathbb{E}\left(\hat{d}\left(X^{n}, Y^{n}, g\left(f_{1}\left(X^{n}\right), f_{2}\left(Y^{n}\right)\right)\right)\right) \leq \Delta .
$$

Definition 2. We say that a tuple $\left(R_{1}, R_{2}, D\right)$ is achievable if $\forall \epsilon>0, \exists$ for all sufficiently large $n$ a transmission system with parameters $\left(n, \theta_{1}, \theta_{2}, \Delta\right)$ such that

$$
\frac{1}{n} \log \theta_{i} \leq R_{i}+\epsilon \quad \text { for } i=1,2 \quad \Delta \leq D+\epsilon .
$$

The performance limit is given by the optimal rate-distortion region $\mathcal{R D}$ which is defined as the set of all achievable tuples $\left(R_{1}, R_{2}, D\right)$.

Note that this problem formulation is very general. For example, defining the joint distortion measure $d(X, Y, \hat{Z})$ as $d_{1}(F(X, Y), \hat{Z})$ enables us to consider the problem of lossy reconstruction of a function of the sources as a special case. Though we only consider a single distortion measure in this paper, it is straightforward to extend the results that we present here for the case of multiple distortion criteria. This implies that the problem of reconstructing the sources subject to two independent distortion criteria (the BergerTung problem [12]) can be subsumed in this formulation with multiple distortion criteria. The Slepian-Wolf [19] problem, the Wyner-Ziv problem [10], the Yeung-Berger problem [13] and the problem of coding with partial side information [7], [8] can also be subsumed by this formulation since they all are special cases of the Berger-Tung problem. The problem of remote distributed source coding [3], [9], where the encoders observe the sources through noisy channels, can also be subsumed in this formulation using the techniques of [14], [15]. We shall see that our coding theorem has implications on the tightness of the Berger-Tung inner bound [12]. The two-user function computation problem of lossy reconstruction of $Z=F(X, Y)$ can also be viewed as a special case of three-user BergerTung problem of encoding the correlated sources $(X, Y, Z)$ with three independent distortion criteria, where the rate of the third encoder is set to zero and the distortions of the first two sources are set to their maximum values.

An achievable rate region for the problem defined in Definitions 1 and 2 can be obtained based on the Berger-Tung coding scheme [12] as follows. Let $\mathcal{P}$ denote the family of pair of conditional probabilities $\left(P_{U \mid X}, P_{V \mid Y}\right)$ defined on $\mathcal{X} \times \mathcal{U}$ and $\mathcal{Y} \times \mathcal{V}$, where $U$ and $V$ are finite sets. For any $\left(P_{U \mid X}, P_{V \mid Y}\right) \in \mathcal{P}$, let the induced joint distribution be $P_{X Y U V}=P_{X Y} P_{U \mid X} P_{V \mid Y} . U, V$ play the role of auxiliary random variables. Define $G: \mathcal{U} \times \mathcal{V} \rightarrow \hat{\mathcal{Z}}$ as that function of $U, V$ that gives the optimal reconstruction $\hat{Z}$ with respect to the distortion measure $d(\cdot, \cdot, \cdot)$. With these definitions, an achievable rate region for this problem is presented below.

Fact 1. For a given source $(X, Y)$ and distortion $d(\cdot, \cdot, \cdot)$ define the region $\mathcal{R} \mathcal{D}_{B T}$ as

$$
\mathcal{R D}_{B T} \triangleq \bigcup_{\left(P_{U \mid X}, P_{V \mid Y}\right) \in \mathcal{P}}\left\{\begin{array}{l}
R_{1} \geq I(X ; U \mid V) \\
R_{2} \geq I(Y ; V \mid U) \\
R_{1}+R_{2} \geq I(X Y ; U V) \\
\mathbb{E}\{d(X, Y, G(U, V))\} \leq D
\end{array}\right\}
$$

Then any $\left(R_{1}, R_{2}, D\right) \in \mathcal{R D}_{B T}^{*}$ is achievable where * denotes convex closure. 
Proof: Follows from the analysis of the Berger-Tung problem [12] in a straightforward way.

\section{DeFINITIONS}

Our coding scheme relies on "embedding" the optimal bivariate function of the auxiliary random variables, $G(U, V)$ in some Abelian group. The idea is to map the two random variables $U \in \mathcal{U}$ and $V \in \mathcal{V}$ to random variables $\tilde{U}$ and $\tilde{V}$ taking values from the Abelian group $\mathbf{G}$ such that the knowledge of $\tilde{U}+\tilde{V}$ is sufficient to know the value of $G(U, V)$. We define the notion of embedding more rigorously in the following:

Definition 3. A bivariate function $G: \mathcal{U} \times \mathcal{V} \rightarrow \mathcal{G}$ is said to be embeddable in an Abelian group $\mathbf{G}$ with respect to the distribution $p_{U V}(u, v)$ on $\mathcal{U} \times \mathcal{V}$ if there exist injective functions $f_{1}: \mathcal{U} \rightarrow \mathbf{G}$ and $f_{2}: \mathcal{V} \rightarrow \mathbf{G}$ and a surjective function $g: \mathbf{G} \rightarrow \mathcal{G}$ such that for all $(u, v) \in \mathcal{U} \times \mathcal{V}$ with $p_{U V}(u, v)>0$ :

$$
g\left(f_{1}(u)+f_{2}(v)\right)=G(u, v)
$$

where the addition is the operation of the group $\mathbf{G}$.

Define the mapped random variables $\tilde{U}=f_{1}(U)$ and $\tilde{V}=f_{2}(V)$. Their dependence on $\mathbf{G}$ is suppressed and the group in question will be clear from the context. The encoding operation is as follows: we reconstruct the function $G(U, V)$ by first embedding it in some Abelian group $\mathbf{G}$ and then reconstructing $\tilde{U}+\tilde{V}$. We define the concepts of group codes and what it means for group codes to be "good" in the following series of definitions.

Definition 4. Let $\mathbf{G}$ be a finite Abelian group. A group code $\mathbb{C}$ of block length $n$ over the group $\mathbf{G}$ is a subset of $\mathbf{G}^{n}$ which is closed under the group addition operation, i.e., $\mathbb{C} \subset \mathbf{G}^{n}$ is such that if $c_{1}, c_{2} \in \mathcal{C}$, then also $c_{1}+c_{2} \in \mathbb{C}$.

Recall that for any group $J$, the kernel $\operatorname{ker}(\phi)$ of a homomorphism $\phi: \mathbf{G}^{n} \rightarrow J$ is a subgroup of $\mathbf{G}^{n}$. We use this fact to build group codes. Given an Abelian group $\mathbf{G} \cong \bigoplus_{i=1}^{I} \mathbb{Z}_{p_{i}^{r_{i}}}$ we construct Abelian group codes of length $n$ over $\mathbf{G}$ as follows

$$
\mathbb{C}=\left\{u \in \mathbf{G}^{n} \mid \phi(u)=c\right\}
$$

where $\phi: \mathbf{G}^{n} \rightarrow \bigoplus_{i=1}^{I} \mathbb{Z}_{p_{i}^{r_{i}}}^{\omega_{i} k}$ is a homomorphism, $\omega_{i}$ 's are a set of weights adding up to one and $k$ is a positive integer. We will justify the use of this ensemble in a more complete version of this work.

We employ nested group codes in our coding scheme. In the distributed source coding problem, we often need one of the components of a nested code to be a good source code while the other one to be a good channel code. We define nested group codes and the notions of "goodness" used to classify a group code as a good source or channel code in the following.

Definition 5. A nested group code consists of two group codes, the inner code (or the coarse code) $\mathbb{C}_{i}$ and the outer code (or the fine code) $\mathbb{C}_{o}$ such that the inner code is a subset (and hence a subgroup) of the outer code $\left(\mathbb{C}_{i} \leq \mathbb{C}_{o}\right)$. We denote the corresponding nested group code by the pair $\left(\mathbb{C}_{i}, \mathbb{C}_{o}\right)$.

When nested group codes are used in distributed source coding, typically the coset leaders of the inner code $\mathbb{C}_{i}$ in the outer code $\mathbb{C}_{o}$ are employed as codewords.

\section{The Group Mutual Information AND the Group ENTROPY}

In this section we define some fundamental quantities related to group codes which appear in the rate region for the distributed source coding problem.

Definition 6. Let $(\mathcal{X}, \mathcal{Y}, W)$ denote a channel with input alphabet $\mathcal{X}$, output alphabet $\mathcal{Y}$ and conditional probabilities $W(y \mid x)$ for $x \in \mathcal{X}$ and $y \in \mathcal{Y}$.

Definition 7. Let $\left(\mathcal{X}, \mathcal{U}, p_{X}, d\right)$ denote a source with source alphabet $\mathcal{X}$, reconstruction alphabet $\mathcal{U}$, source distribution $p_{X}$ and distortion measure $d: \mathcal{X} \times \mathcal{U} \rightarrow \mathbb{R}^{+}$.

\section{A. Channel Coding: The $\mathbb{Z}_{p^{r}}$ Case}

For the channel $(\mathcal{X}, \mathcal{Y}, W)$, let $X$ be a random variable taking values from $\mathcal{X}=\mathbf{G}=\mathbb{Z}_{p^{r}}$ and $Y$ be the corresponding channel output. For a subgroup $H_{\theta}=p^{\theta} \mathbb{Z}_{p^{r}}$ of $\mathbf{G}$, define the random variable $[X]_{\theta}$ taking values from cosets of $H_{\theta}$ in $\mathbf{G}$ as

$$
[X]_{\theta}=X+H_{\theta}
$$

The channel coding rate of group codes over $\mathbf{G}=\mathbb{Z}_{p^{r}}$ for the channel is defined as

$$
\begin{aligned}
\bar{I}_{c . c .}^{\mathrm{G}}(X ; Y) & =\min _{\theta=0}^{r-1}\left[r \log p-\frac{r}{(r-\theta)} H\left(X \mid Y[X]_{\theta}\right)\right] \\
& =\min _{\theta=0}^{r-1} \frac{r}{(r-\theta)} I\left(X ; Y \mid[X]_{\theta}\right)
\end{aligned}
$$

\section{B. Source Coding: The $\mathbb{Z}_{p^{r}}$ Case}

For a source $\left(\mathcal{X}, \mathcal{U}, p_{X}, d\right)$, Let $U$ be a random variable taking values from $\mathcal{U}=\mathbf{G}=\mathbb{Z}_{p^{r}}$ and let $X$ be a random variable distributed over $\mathcal{X}$ according to $p_{X}$. Assume $U$ is jointly distributed with $X$ such that $\mathbb{E}\{d(X, U)\}<D$. For a subgroup $H_{\theta}=p^{\theta} \mathbb{Z}_{p^{r}}$ of $\mathbf{G}$, define the random variable $[U]_{\theta}$ taking values from cosets of $H_{\theta}$ in $\mathbf{G}$ as

$$
[U]_{\theta}=U+H_{\theta}
$$

The source coding rate of group codes over $\mathbf{G}=\mathbb{Z}_{p^{r}}$ for the test channel $p_{X \mid U}$ is defined as

$$
\begin{aligned}
\bar{I}_{s . c .}^{\mathbf{G}}(U ; X) & =\max _{\theta=1}^{r}\left[r \log p-\frac{r}{\theta} H\left([U]_{\theta} \mid X\right)\right] \\
& =\max _{\theta=1}^{r} \frac{r}{\theta} I\left([U]_{\theta} ; X\right)
\end{aligned}
$$




\section{Channel Coding: The General Case}

Any Abelian group $\mathbf{G}$ can be decomposed as

$$
\mathbf{G}=\bigoplus_{i=1}^{I} \mathbb{Z}_{p_{i}^{r_{i}}}
$$

where for $i=1, \cdots, I, p_{i}$ is a prime and $r_{i}$ is a positive integer. For the channel $(\mathcal{X}, \mathcal{Y}, W)$, let $X$ be a random variable taking values from $\mathcal{X}=\mathbf{G}$ and $Y$ be the corresponding channel output. Let $\theta=\left(\theta_{1}, \cdots, \theta_{I}\right)$ be such that $0 \leq \theta_{i} \leq r_{i}$ for $i=1, \cdots, I$ and let $H$ be a subgroup of $\mathbf{G}$ defined by,

$$
H=\bigoplus_{i=1}^{I} p_{i}^{\theta_{i}} \mathbb{Z}_{p_{i}^{r_{i}}}
$$

If a subgroup $H$ of $\mathbf{G}$ can be written in this form for some $\theta$, we denote this by $H \preceq \mathbf{G}$. Define the random variable $[X]_{H}$ taking values from cosets of $H$ in $\mathbf{G}$ as

$$
[X]_{H}=X+H
$$

The channel coding rate of group codes over $\mathbf{G}$ for the channel is defined as

$$
\bar{I}_{\text {c.c. }}^{\mathbf{G}}(X ; Y)=\max _{\substack{w_{1}, \cdots, w_{I} \\ w_{1}+\cdots+w_{I}=1}} \min _{\substack{H \prec \mathbf{G} \\ H \neq 0}} \frac{1}{w_{H}} I\left(X ; Y \mid[X]_{H}\right)
$$

where

$$
w_{H}=\sum_{i=1}^{I} \frac{r_{i}-\theta_{i}}{r_{i}} w_{i}
$$

Note that this definition is a generalization of the definition made in (6). When $\mathbf{G}$ is a field of prime order, $\mathbf{G}=\mathbb{Z}_{p}$, both definitions coincide with the definition of the symmetric capacity of the channel.

\section{Source Coding: The General Case}

Let $\mathbf{G}$ be the group defined in (8). For a source $\left(\mathcal{X}, \mathcal{U}, p_{X}, d\right)$, Let $U$ be a random variable over $\mathcal{U}=\mathbf{G}$ and let $X$ be a random variable distributed over $\mathcal{X}$ according to $p_{X}$. Assume $U$ is jointly distributed with $X$ such that $\mathbb{E}\{d(X, U)\}<D$. Let $\theta=\left(\theta_{1}, \cdots, \theta_{I}\right)$ be such that $0 \leq \theta_{i} \leq r_{i}$ for $i=1, \cdots, I$ and let $H$ be a subgroup of G defined by (9). Define the random variable $[U]_{H}$ taking values from cosets of $H$ in $\mathbf{G}$ as $[U]_{H}=U+H$. The source coding rate of group codes over $\mathbf{G}$ for the test channel $p_{X \mid U}$ is defined as

$$
\bar{I}_{\text {s.c. }}^{\mathbf{G}}(U ; X)=\min _{\substack{w_{1}, \cdots, w_{I} \\ w_{1}+\cdots+w_{I}=1}} \max _{\substack{H \prec G \\ H \neq \mathbf{G}}} \frac{1}{1-w_{H}} I\left([U]_{H} ; X\right)
$$

where $w_{H}$ is defined in (11). This definition is a generalization of the definition made in (7). When $\mathbf{G}$ is a field of prime order, $\mathbf{G}=\mathbb{Z}_{p}$, both definitions coincide with the definition of the symmetric capacity of the test channel $p_{X \mid U}$.
For the source and channel models define as above, define the channel coding group entropies of the group $\mathbf{G}$ as

$$
\begin{aligned}
& \bar{H}_{\text {c.c. }}^{\mathbf{G}}(X \mid Y)=\log |\mathbf{G}|-\bar{I}_{\text {c.c. }}^{\mathbf{G}}(X ; Y) \\
& \bar{H}_{c . c .}^{\mathbf{G}}(X)=\bar{H}_{\text {c.c. }}^{\mathbf{G}}(X \mid 0)
\end{aligned}
$$

and the source coding group entropies of the group $\mathbf{G}$ as

$$
\begin{aligned}
& \bar{H}_{\text {s.c. }}^{\mathbf{G}}(U \mid X)=\log |\mathbf{G}|-\bar{I}_{\text {s.c. }}^{\mathbf{G}}(U ; X) \\
& \bar{H}_{\text {s.c. }}^{\mathbf{G}}(U)=\bar{H}_{\text {s.c. }}^{\mathbf{G}}(U \mid 0)
\end{aligned}
$$

It has been shown in [17] that the rate $R=\bar{I}_{c . c .}^{\mathbf{G}}(X ; Y)$ is achievable using group codes for lossless transmission of information over the channel $\left(\mathbf{G}, \mathcal{Y}, W_{Y \mid X}\right)$ where $X$ is uniform. Similarly, the rate $R=\bar{I}_{\text {s.c. }}^{\mathbf{G}}(U ; S)$ is achievable using group codes over $\mathbf{G}$ for the lossy compression of the source $\left(\mathcal{X}, \mathbf{G}, p_{X}, d\right)$ with a distortion $D$ if $U$ satisfies the condition $\mathbb{E}\{d(X, U)\}<D$ and $U$ is uniform. It can also be shown that the rate $\bar{I}_{s . c .}^{\mathbf{G}}(U ; X)-\bar{I}_{c . c .}^{\mathbf{G}}(U ; S)$ is achievable using nested group codes for the source coding problem with side information $S$ available at the decoder.

\section{Decoding the Sum of two Distributed Sources}

Consider two correlated source $U$ and $V$ taking valued from sets $\mathcal{U}=\mathbf{G}$ and $\mathcal{V}=\mathbf{G}$ respectively where $\mathbf{G}$ is an Abelian group. In a distributed source coding setting, assume the $X$ and the $Y$-encoders have access to the source sequences $X^{n}$ and $Y^{n}$ respectively and the joint decoder is required to reconstruct the sum $Z^{n}=X^{n}+Y^{n}$ where the addition is the group operation. This problem has been studied by Ahlswede and Han in [16] for the case where all random variables are binary $\left(\mathbf{G}=\mathbb{Z}_{2}\right)$. An achievable rate region for this problem for the binary case is as follows:

Let $P$ and $Q$ be two finite random variables satisfying the following Markov chain:

$$
P \rightarrow U \rightarrow V \rightarrow Q
$$

Then the rate pair $\left(R_{1}, R_{2}\right)$ is achievable if:

$$
\begin{aligned}
& R_{1} \geq I(P ; U \mid Q)+H(Z \mid P Q) \\
& R_{2} \geq I(Q ; V \mid P)+H(Z \mid P Q) \\
& R_{1}+R_{2} \geq I(P Q ; U V)+2 H(Z \mid P Q)
\end{aligned}
$$

Here, we state the result for the general case where $U, V$ and $Z$ take values from an arbitrary Abelian group G: Let $P$ and $Q$ be two finite random variables satisfying the following Markov chain:

$$
P \rightarrow U \rightarrow V \rightarrow Q
$$

Then the following corner points are achievable:

$$
\left\{\begin{array}{l}
R_{1}=I(P ; U)+\bar{H}_{c . c}^{\mathrm{G}}(Z \mid P Q) \\
R_{2}=I(Q ; V \mid P)+\bar{H}_{c . c .}^{\mathrm{G}}(Z \mid P Q)
\end{array}\right.
$$

and

$$
\left\{\begin{array}{l}
R_{1}=I(P ; U \mid Q)+\bar{H}_{c . c}^{\mathbf{G}}(Z \mid P Q) \\
R_{2}=I(Q ; V)+\bar{H}_{c . c .}^{\mathbf{G}}(Z \mid P Q)
\end{array}\right.
$$

This rate region is equivalent to the rate region provided in [16] when the random variables are binary. 


\section{Nested Group Codes For THE Wyner-Ziv PROBLEM}

Let $\mathbf{G}$ be the group defined in (8). In this section we use nested Abelian group codes over $\mathbf{G}$ to find an achievable rate region for the Wyner-Ziv problem. The proofs are omitted and will be available in a more complete version of this work. Assume the source of information $X$ takes values from the set $\mathcal{X}$ and is jointly distributed with the side information $S$ with distribution $p_{X S}$. Let $\hat{\mathcal{X}}$ be the reconstruction alphabet and let $d: \mathcal{X} \times \hat{\mathcal{X}} \rightarrow \mathbb{R}^{+}$be the distortion criterion. Let $U$ be a random variable taking values from the set $\mathcal{U}=\mathbf{G}$ jointly distributed with $X$ and $S$ according to $p_{X S} p_{U \mid X}$. Further assume that there exists a function $f: \mathcal{S} \times \mathcal{U} \rightarrow \hat{\mathcal{X}}$ such that $\mathbb{E}\{d(X, f(S, U))\} \leq D$. Assume that the side information is available to the receiver.

We construct the ensemble of nested group codes as the set of all $\left(\mathbb{C}_{i}, \mathbb{C}_{o}\right)$ where

$$
\begin{aligned}
& \mathbb{C}_{o}=\left\{u \in \mathbf{G}^{n} \mid \phi(u)=c\right\} \\
& \mathbb{C}_{i}=\left\{u \in \mathbf{G}^{n} \mid \phi(u)=c, \Delta \phi(u)=\Delta c\right\}
\end{aligned}
$$

where $\phi$ is a homomorphism from $\mathbf{G}^{n}$ to the group $J, \Delta \phi$ is a homomorphism from $\mathbf{G}^{n}$ to the group $\Delta J, c$ is a vector in $J$ and $\Delta c$ is a vector in $\Delta J$ where

$$
\begin{gathered}
J=\bigoplus_{i=1}^{I} \mathbb{Z}_{p_{i}^{r_{i}}}^{w_{i} k} \\
\Delta J=\bigoplus_{i=1}^{I} \mathbb{Z}_{p_{i}^{r_{i}}}^{w_{r_{i}}}
\end{gathered}
$$

for some positive integers $k$ and $l$. Note that the rate of the outer code is given by

$$
R_{o}=\log |\mathbf{G}|-\frac{k}{n} \sum_{i=1}^{I} w_{i} r_{i} \log p_{i}
$$

and the rate of the inner code is equal to

$$
R_{i}=\log |\mathbf{G}|-\frac{k+l}{n} \sum_{i=1}^{I} w_{i} r_{i} \log p_{i}
$$

Hence, the rate of transmission is equal to

$$
\begin{aligned}
R & =R_{o}-R_{i} \\
& =\frac{l}{n} \sum_{i=1}^{I} w_{i} r_{i} \log p_{i}
\end{aligned}
$$

The encoding rule is as follows: Given a source output $x \in$ $\mathcal{X}^{n}$, if $x$ is typical with respect to the source distribution, look for a codeword $u$ in the outer code $\left(u \in \mathbb{C}_{o}\right)$ such that $u$ is jointly typical with $x\left(u \in A_{\epsilon}^{n}(U \mid x)\right)$ with respect to $p_{X U}$. If $x$ is not typical or if there does not exist such a codeword $u$ declare error. If no encoding error occurs, send $\Delta \phi(u)$ to the receiver.

At the receiver, given the side information $s \in \mathcal{S}^{n}$ and $\Delta \phi(u)$, look for a codeword $\hat{u} \in \mathbb{C}_{o}$ which is in the same coset of $\mathbb{C}_{i}$ as $u(\Delta \phi(\hat{u})=\Delta \phi(u))$ and such that $\hat{u} \in A_{\epsilon}^{n}(U \mid s)$. If there does not exist such a $\hat{u}$ declare error.

In order for the encoding error to go to zero we require the following condition:

$$
R_{o} \geq \bar{I}_{\text {s.c. }}^{\mathrm{G}}(U ; X)
$$

and in order for the decoding error to go to zero we require:

$$
R_{i} \leq \bar{I}_{c . c .}^{\mathbf{G}}(U ; S)
$$

Therefore, the transmission rate can be arbitrarily close to $R=\bar{I}_{s . c .}^{\mathbf{G}}(U ; X)-\bar{I}_{c . c .}^{\mathbf{G}}(U ; S)$.

Note that for the case where the underlying group is a field of prime size $\mathbf{G}=\mathbb{Z}_{p}$ the we have

$$
\begin{aligned}
R & =\bar{I}_{s . c .}^{\mathbf{G}}(U ; X)-\bar{I}_{c . c .}^{\mathbf{G}}(U ; S) \\
& =\log p-H(U \mid X)-(\log p-H(U \mid S)) \\
& =I(U ; X)-I(U ; S)
\end{aligned}
$$

Hence there is no loss in the rate for this case.

\section{Nested Group Codes for Distributed Source CODING}

In this section we use nested Abelian group codes over $\mathbf{G}$ to find an achievable rate region for the distributed source coding problem. The proofs are omitted and will be available in a more complete version of this work. Let $X, Y, U, V, Z$ be five random variables where $X$ and $Y$ are two distributed sources jointly distributed according to $p_{X Y}$ and $U$ and $V$ are auxiliary random variables taking values from the group G. Let $U \rightarrow X \rightarrow Y \rightarrow V$ form a Markov chain and let $Z=U+V$ where + is the group operation. In this section, we analyze the performance of nested group codes for the problem of reconstructing $Z$ in this distributed source coding problem. We use two nested group codes; one for each encoder. Let $\left(\mathbb{C}_{i}, \mathbb{C}_{o 1}\right)$ be the nested group code employed by the $X$-encoder where

$$
\begin{aligned}
& \mathbb{C}_{o 1}=\left\{u \in \mathbf{G}^{n} \mid \phi_{2}(u)=c_{2}\right\} \\
& \mathbb{C}_{i}=\left\{u \in \mathbf{G}^{n} \mid \phi_{1}(u)=c_{1}, \phi_{2}(u)=c_{2}, \Delta \phi(u)=\Delta c\right\}
\end{aligned}
$$

and let $\left(\mathbb{C}_{i}, \mathbb{C}_{o 2}\right)$ be the nested group code employed by the $Y$-encoder where

$$
\begin{aligned}
& \mathbb{C}_{o 2}=\left\{v \in \mathbf{G}^{n} \mid \phi_{1}(v)=c_{1}\right\} \\
& \mathbb{C}_{i}=\left\{v \in \mathbf{G}^{n} \mid \phi_{1}(v)=c_{1}, \phi_{2}(v)=c_{2}, \Delta \phi(v)=\Delta c\right\}
\end{aligned}
$$

where $\phi_{1}$ is a homomorphism from $\mathbf{G}^{n}$ to the group $J_{1}$, $\phi_{2}$ is a homomorphism from $\mathbf{G}^{n}$ to the group $J_{2}, \Delta \phi$ is a homomorphism from $\mathbf{G}^{n}$ to the group $\Delta J, c_{1}$ is a vector in 
$J_{1}, c_{2}$ is a vector in $J_{2}$, and $\Delta c$ is a vector in $\Delta J$ where

$$
\begin{aligned}
& J_{1}=\bigoplus_{i=1}^{I} \mathbb{Z}_{p_{i}^{r_{i}}}^{w_{k_{1}} k_{1}} \\
& J_{2}=\bigoplus_{i=1}^{I} \mathbb{Z}_{p_{i}^{r_{i}}}^{w_{i_{i} k_{2}}} \\
& \Delta J=\bigoplus_{i=1}^{I} \mathbb{Z}_{p_{i}^{r_{i}}}^{w_{i_{i} l}}
\end{aligned}
$$

for some positive integers $k_{1}, k_{2}$ and $l$. Note that the two nested group codes share the same inner code but may have different outer codes. The rate of the code employed by the $X$-encoder is given by

$$
R_{1}=\frac{k_{1}+l}{n} \sum_{i=1}^{I} w_{i} r_{i} \log p_{i}
$$

and the rate of the $Y$-encoder is equal to

$$
R_{2}=\frac{k_{2}+l}{n} \sum_{i=1}^{I} w_{i} r_{i} \log p_{i}
$$

The encoding rule for the $X$-encoder is as follows: Given a source output $x \in \mathcal{X}^{n}$, if $x$ is typical with respect to the source distribution, look for a codeword $u$ in the outer code $\left(u \in \mathbb{C}_{o 1}\right)$ such that $u$ is jointly typical with $x\left(u \in A_{\epsilon}^{n}(U \mid x)\right)$ with respect to $p_{X U}$. If $x$ is not typical or if there does not exist such a codeword $u$ declare error. If no encoding error occurs, send $\phi_{1}(u)$ and $\Delta \phi(u)$ to the receiver.

The encoding rule for the $Y$-encoder is similar: Given a source output $y \in \mathcal{Y}^{n}$, if $y$ is typical with respect to the source distribution, look for a codeword $v$ in the outer code $\left(v \in \mathbb{C}_{o 2}\right)$ such that $v$ is jointly typical with $y\left(v \in A_{\epsilon}^{n}(V \mid y)\right)$ with respect to $p_{Y V}$. If $y$ is not typical or if there does not exist such a codeword $v$ declare error. If no encoding error occurs, send $\phi_{2}(v)$ and $\Delta \phi(v)$ to the receiver.

At the receiver, after receiving $\phi_{1}(u), \Delta \phi(u), \phi_{2}(v)$ and $\Delta \phi(v)$, look for a typical vector $\hat{z} \in \mathbf{G}^{n}$ such that

$$
\begin{aligned}
& \phi_{1}(\hat{z})=\phi_{1}(u)+\phi_{1}(v)=\phi_{1}(u)+c_{1} \\
& \phi_{2}(\hat{z})=\phi_{2}(u)+\phi_{2}(v)=c_{2}+\phi_{2}(v) \\
& \Delta \phi(\hat{z})=\Delta \phi(u)+\Delta \phi(v)
\end{aligned}
$$

In order for the encoding errors to go to zero we require the following conditions:

$$
\begin{aligned}
& R_{o 1} \geq \bar{I}_{s . c .}^{\mathbf{G}}(U ; X)=\log |\mathbf{G}|-\bar{H}_{s . c .}^{\mathbf{G}}(U \mid X) \\
& R_{o 2} \geq \bar{I}_{s . c .}^{\mathbf{G}}(V ; Y)=\log |\mathbf{G}|-\bar{H}_{s . c .}^{\mathbf{G}}(V \mid Y)
\end{aligned}
$$

where $R_{o 1}$ is the rate of the outer code corresponding to the $X$-encoder and $R_{o 2}$ is the rate of the outer code corresponding to the $Y$-encoder. In order for the decoding error to go to zero we require:

$$
R_{i} \leq \log |\mathbf{G}|-\bar{H}_{c . c .}^{\mathbf{G}}(Z)
$$

Therefore, the transmission rates can be arbitrarily close to

$$
\begin{aligned}
& R_{1}=\bar{H}_{c . c .}^{\mathbf{G}}(Z)-\bar{H}_{s . c .}^{\mathbf{G}}(U \mid X) \\
& R_{2}=\bar{H}_{c . c .}^{\mathbf{G}}(Z)-\bar{H}_{s . c .}^{\mathbf{G}}(V \mid Y)
\end{aligned}
$$

\section{THE CODING THEOREM}

We are given discrete random variables $X$ and $Y$ which are jointly distributed according to $P_{X Y}$. Let $\mathcal{P}$ denote the family of pair of conditional probabilities $\left(P_{U \mid X}, P_{V \mid Y}\right)$ defined on $\mathcal{X} \times \mathcal{U}$ and $\mathcal{Y} \times \mathcal{V}$, where $\mathcal{U}$ and $\mathcal{V}$ are finite sets, $|\mathcal{U}|=$ $\alpha,|\mathcal{V}|=\beta$. For any $\left(P_{U \mid X}, P_{V \mid Y}\right) \in \mathcal{P}$, let the induced joint distribution be $P_{X Y U V}=P_{X Y} P_{U \mid X} P_{V \mid Y} . U, V$ play the role of auxiliary random variables. Define $G: \mathcal{U} \times \mathcal{V} \rightarrow \hat{\mathcal{Z}}$ as that function of $U, V$ that gives the optimal reconstruction $\hat{Z}$ with respect to the distortion measure $d(\cdot, \cdot, \cdot)$. Let $\mathcal{G}$ denote the image of $G(U, V)$. Let $\mathcal{T}=\{\mathbf{G}: \mathbf{G}$ is Abelian, $|\mathcal{G}| \leq|\mathbf{G}| \leq$ $\alpha \beta, G(U, V) \subset \mathbf{G}$ with respect to $\left.P_{U V}\right\}$. Let $\tilde{U}=f_{1}(U)$ and $\tilde{V}=f_{2}(V)$ where the mappings are as defined in Definition 3. Let $P$ and $Q$ be two finite random variables satisfying the following Markov chain:

$$
P U \rightarrow X \rightarrow Y \rightarrow Q V
$$

With these definitions, an achievable rate region is presented below:

Theorem 1. For a given source $(X, Y)$, the rate pair $\left(R_{1}, R_{2}\right)$ is achievable if:

$$
\left\{\begin{array}{c}
R_{1} \geq I(X ; P \mid Q)+\bar{H}_{c . c .}^{\mathbf{G}}(\tilde{Z} \mid P Q)-\bar{H}_{s . c .}^{\mathbf{G}}(\tilde{U} \mid X Q) \\
R_{2} \geq I(Y ; Q \mid P)+\bar{H}_{c . c .}^{\mathbf{G}}(\tilde{Z} \mid P Q)-\bar{H}_{\text {s.c. }}^{\mathbf{G}}(\tilde{V} \mid Y P) \\
R_{1}+R_{2} \geq I(X Y ; P Q)+2 \bar{H}_{c . c .}^{\mathbf{G}}(\tilde{Z} \mid P Q) \\
-\bar{H}_{\text {s.c. }}^{\mathbf{G}}(\tilde{U} \tilde{V} \mid X Y P Q)
\end{array}\right.
$$

Since the encoders don't communicate with each other, we impose the Markov chain $P V-Y-X-Q U$ on the joint distribution $P_{X Y U V}$. The family $\mathcal{P}$ contains all distributions that satisfy this Markov chain. Fix such a joint distribution. The encoding is performed in two stages: In the first stage $P$ and $Q$ are encoded using random codes so that the $X$ encoder expends a rate of $I(X ; P)$ and the $Y$-encoder expends a rate of $I(Y ; Q \mid P)$. In the second stage, nested group codes are employed in the manner described in Section VII, so that the $X$-encoder expends an additional rate of $\bar{H}_{c . c .}^{\mathbf{G}}(\tilde{Z} \mid P Q)-$ $\bar{H}_{s . c .}^{\mathbf{G}}(\tilde{U} \mid X P Q)$ and the $Y$-encoder expends an additional rate of $\bar{H}_{c . c .}^{\mathbf{G}}(\tilde{Z} \mid P Q)-\bar{H}_{s . c .}^{\mathbf{G}}(\tilde{V} \mid Y P Q)$. Therefore, the following rate pair is achievable:

$$
\begin{aligned}
& R_{1}=I(X ; P)+\bar{H}_{c . c .}^{\mathbf{G}}(\tilde{Z} \mid P Q)-\bar{H}_{s . c .}^{\mathbf{G}}(\tilde{U} \mid X Q) \\
& R_{2}=I(Y ; Q \mid P)+\bar{H}_{c . c .}^{\mathbf{G}}(\tilde{Z} \mid P Q)-\bar{H}_{s . c .}^{\mathbf{G}}(\tilde{V} \mid Y P)
\end{aligned}
$$

In a similar fashion, we can show that the following rate pair is achievable:

$$
\begin{aligned}
& R_{1}=I(X ; P \mid Q)+\bar{H}_{c . c .}^{\mathbf{G}}(\tilde{Z} \mid P Q)-\bar{H}_{s . c .}^{\mathbf{G}}(\tilde{U} \mid X Q) \\
& R_{2}=I(Y ; Q)+\bar{H}_{c . c .}^{\mathbf{G}}(\tilde{Z} \mid P Q)-\bar{H}_{s . c .}^{\mathbf{G}}(\tilde{V} \mid Y P)
\end{aligned}
$$

This proves the achievability of the rate region given in (17). 
Remark 1: The above coding theorem can be extended to the case of multiple distortion constraints in a straightforward fashion.

\section{ImPRovement Using RANDOM/GRoup CODES}

Note that the only property of the structured codes which is used in our coding scheme is the fact that the two inner codes are closed under addition and we do not require the outer code to be a group code. In this section, we use this observation and consider the ensemble of codes constructed in the following manner. The inner codes are constructed similar to the previous section (constructed as group codes). However, the outer code is constructed as a random union of several cosets of the inner code. Hence the outer code is not necessarily closed under addition (and it is not required to be). The inner code is defined as

$$
\mathbb{C}_{i}=\left\{u \in \mathbf{G}^{n} \mid \phi(u)=c\right\}
$$

where for some weights $w_{i}$ adding up to one and a positive integer $k, \phi$ is a homomorphism from $\mathbf{G}^{n}$ to $J=\bigoplus_{i=1}^{I} \mathbb{Z}_{p_{i}^{i_{i}}}^{k w_{i}}$. The outer code is defined as

$$
\mathbb{C}_{i}=\left\{u \in \mathbf{G}^{n} \mid \phi(u)=c_{i} \text { for some } i=1, \cdots, 2^{\alpha n}\right\}
$$

for some positive number $\alpha$ and vectors $c_{i} \in J, i=$ $1, \cdots, 2^{\alpha n}$. The achievable rate region using this new ensemble is provided in the following: Define the random variables $X, Y, U, V, \tilde{U}, \tilde{V}, P, Q$ as in Section VIII.

Theorem 2. For the correlated sources $(X, Y)$ and the random variable defined above, the rate pair $\left(R_{1}, R_{2}\right)$ is achievable if:

$$
\left\{\begin{array}{c}
R_{1} \geq I(X ; P \mid Q)+\bar{H}_{\text {c.c. }}^{\mathrm{G}}(\tilde{Z} \mid P Q)-H(\tilde{U} \mid X Q) \\
R_{2} \geq I(Y ; Q \mid P)+\bar{H}_{\text {c.c. }}^{\mathrm{G}}(\tilde{Z} \mid P Q)-H(\tilde{V} \mid Y P) \\
R_{1}+R_{2} \geq I(X Y ; P Q)+2 \bar{H}_{c . c .}^{\mathrm{G}}(\tilde{Z} \mid P Q) \\
-H(\tilde{U} \tilde{V} \mid X Y P Q)
\end{array}\right.
$$

Note that source coding group entropies are always greater than or equal to the ordinary entropies and hence this achievable rate region contains the achievable rate region given in (17).

\section{COMPARISON}

It has been shown in [1] that the provided achievable rate region is strictly larger than other known results. Compared to the rate region provided in [1], the new rate region presented in this paper benefits from two improvements in the coding scheme. First, the outer code in the new scheme does not have to be a group code. Therefore the rate loss incurred in the source coding part of the coding scheme provided in [1] has been removed. A second improvement of our coding scheme is that the decoding operation is done jointly as compares to successive decoding used in [1].

\section{CONCLUSion}

We used structured codes constructed over Abelian groups for the distributed source coding problem and provided a new inner bound on their performance limit. Specifically, we used nested Abelian group codes and nested random/group codes, to achieve a new inner bound for the rate-distortion region. This inner bound contains all the known achievable rate regions and is strictly larger than other known rate regions for certain sources.

\section{REFERENCES}

[1] D. Krithivasan and S. S. Pradhan, "Distributed Source Coding Using Abelian Group Codes," IEEE Trans. Inform. Theory, vol. 57, pp. 14951519, March 2011.

[2] H. Yamamoto and K. Itoh, "Source coding theory for multiterminal communication systems with a remote source," The Transactions of the IECE of Japan, vol. E-63, pp. 700-706, October 1980.

[3] T. J. Flynn and R. M. Gray, "Encoding of correlated observations," IEEE Trans. Inform. Theory, vol. IT-33, pp. 773-787, November 1987.

[4] A. Orlitsky and J. R. Roche, "Coding for computing," IEEE Trans. Inform. Theory, vol. IT-47, pp. 903-917, March 2001.

[5] H. Yamamoto, "Wyner-Ziv theory for a general function of the correlated sources," IEEE Trans. Inform. Theory, vol. IT-28, pp. 803-807, September 1982.

[6] J. Korner and K. Marton, "How to encode the modulo-two sum of binary sources," IEEE Trans. Inform. Theory, vol. IT-25, pp. 219-221, March 1979.

[7] A. D. Wyner, "On source coding with side information at the decoder," IEEE Trans. on Inform. Theory, vol. IT-21, pp. 294-300, May 1975.

[8] R. Ahlswede and J. Korner, "Source coding with side information and a converse for degraded broadcast channels," IEEE Trans. Inform. Theory, vol. IT- 21, pp. 629-637, November 1975.

[9] H. Viswanathan, Z. Zhang, and T. Berger, "The CEO problem," IEEE Trans. on Inform. Theory, vol. 42, pp. 887-902, May 1996.

[10] A. D. Wyner and J. Ziv, "The rate-distortion function for source coding with side information at the decoder," IEEE Trans. Inform. Theory, vol. IT- 22, pp. 1-10, January 1976.

[11] T. Berger, "Multiterminal source coding," in Lectures presented at CISM summer school on the Inform. Theory approach to communications, July 1977.

[12] S.-Y. Tung, Multiterminal source coding. $\mathrm{PhD}$ thesis, School of Electrical Engineering, Cornell University, Ithaca, NY, May 1978.

[13] T. Berger and R. W. Yeung, "Multiterminal source coding with one distortion criterion", IEEE Trans. on Inform. Theory, vol. IT-35, pp. 228236, March 1989.

[14] R. Dobrushin and B. Tsybakov, "Information transmission with additional noise," IRE Trans. Inform. Theory, vol. IT- 18, pp. S293-S304, 1962.

[15] H. S. Witsenhausen, "Indirect rate distortion problems," IEEE Trans. Inform. Theory, vol. IT- 26, pp. 518-521, September 1980.

[16] R. Ahlswede and T. Han, "On Source Coding with Side Information via a Multiple Access Channel and Related Problems in Multi-User Information Theory,' IEEE Trans. Inform. Theory, vol. IT- 29, pp. 396412, September 1983.

[17] A. G. Sahebi, S. S. Pradhan, "On the Capacity of Abelian Group Codes Over Discrete Memoryless Channels", Proc. IEEE International Symposium on Inform. Theory, Saint Petersburg, Russia, 2011.

[18] S. Gelfand and M. Pinsker, "Coding of sources on the basis of observations with incomplete information," Problemy Peredachi Informatsii, vol. 15, pp. 45-57, Apr-June 1979.

[19] D. Slepian and J. K. Wolf, "Noiseless coding of correlated information sources," IEEE Trans. on Inform. Theory, vol. 19, pp. 471-480, July 1973. 